\title{
Spumarétrovirus et maladies auto-immunes
}

Les spumarétrovirus (voir l'article de Verdier et al., p. 536 de ce numéro) constituent l'un des sept groupes de la famille des retroviridae. Le prototype humain est connu sous le nom de HFV (human foamy virus). Premier rétrovirus à avoir été isolé chez l'homme, il présente des similitudes de structure génomique avec les autres rétrovirus humains connus actuellement: les VIH et HTLV. En effet, en plus des gènes structuraux gag, pol et env, son génome comporte des gènes qui codent pour des protéines régulatrices de l'expression virale. Ces protéines peuvent, par ailleurs, agir sur l'expression de gènes cellulaires. Les spumarétrovirus sont responsables, chez leurs hôtes naturels, d'infections chroniques où la persistance virale ne semble pas être accompagnée de signes cliniques particuliers.

Malgré les progrès réalisés ces dernières années en ce qui concerne la virologie générale et la biologie moléculaire des spumarétrovirus, aucune maladie spécifique n'a pu leur être associée de manière définitive [1]. Si la thyroïdite subaiguë de De Quervain a pu être considérée comme une affection probablement due à un spumarétrovirus [2], des travaux récents n'ont pu confirmer cette association [3].

Le rôle des rétrovirus dans l'étiopathologie des maladies auto-immunes nombreux auteurs, mais n'a jamais été clairement confirmé. Ainsi, certains rétrovirus, par leurs propriétés d'induire des infections latentes chez leurs hôtes, pourraient être des éléments déclenchants et/ou de maintien des processus auto-immuns. Dans ce contexte, nous avons recherché d'éventuels marqueurs d'infection par HFV chez des patients souffrant de différentes maladies auto-immunes comme le purpura thrombopénique idiopathique, la maladie de Basedow, la thyroïdite chronique d'Hashimoto et la myasthénie gravis.

Les résultats obtenus montrent clairement qu'aucune trace d'infection par les spumarétrovirus n'a pu être mise en évidence dans les dix-sept cas de purpura thrombopénique idiopathique étudiés. En revanche, la technique d'amplification génique (PCR), utilisant des amplimères spécifiques correspondant aux différents domaines génomiques d'HFV, a permis de déceler des séquences d'ADN apparentées aux spumarétrovirus chez certains patients souffrant des trois autres affections. En effet, cette recherche par PCR dans l'ADN des lymphocytes circulants a été positive chez un nombre significatif de patients atteints de la maladie de Basedow [4]. En revanche, la présence d'anticorps anti-HFV n'a pu être facilement mise en évidence dans le sérum de ces patients. Seule l'application de techniques plus adaptées a conduit à la détection de taux très faibles d'anticorps. En outre, certains malades souffrant de la maladie d'Hashimoto ont également présenté une forte positivité en PCR, mais avec une fréquence moins importante que celle rencontrée chez les patients atteints de maladie de Basedow.

Nous étudions actuellement le statut immunologique de ces endocrinopathies thyroïdiennes vis-à-vis des protéines virales. Plusieurs hypothèses, non exclusives, peuvent être avancées pour expliquer la difficulté à détecter les anticorps anti-HFV chez les patients atteints de la maladie de Basedow. Il se pourrait que toutes les séquences détectées ne soient pas exprimées. Alternativement, la production d'anticorps pourrait être masquée par la formation de complexes immuns rendant difficile leur détection. Finalement, les tests immunologiques utilisés pourraient ne pas être adaptés à la révélation de faibles taux d'anticorps.

Ces résultats et de récents travaux décrivant l'apparition d'une myopathie chez des souris transgéniques ayant intégré le génome de HFV, ou seulement ses gènes régulateurs [5], nous ont poussés à rechercher des marqueurs d'infection chez des patients myasthéniques. Une étude préliminaire, entreprise au sein d'un groupe d'individus souffrant de 
myasthénie gravis, nous a permis de décrire très récemment le cas d'un patient présentant, à la fois, des séquences génomiques de HFV dans l'ADN lymphocytaire, et des anticorps spécifiques dirigés contre certaines protéines virales, révélés par immunoprécipitation [6].

L'existence de marqueurs spumarétroviraux chez des patients atteints de maladies auto-immunes nous ouvre des perspectives intéressantes quant au rôle possible des infections chroniques induites par HFV chez l'homme. Des expériences en cours doivent nous permettre d'élargir nos études à d'autres entités nosologiques, et d'éclaircir les mécanismes d'action de cet agent viral au cours du déséquilibre immunitaire.

A.S.

R.E-R

G.P.

1. Loh PC. Spumaviruses. In : Levy JA Fraenkel-Conrat $\mathrm{H}, \mathrm{W}$ 'agner RR, eds. The Retro viridae. New York: Plenum Press, 1992: 361-97.

2. Stancek D, Stancekova M, Janotka M, Hnilica P, Oravec D. Isolation and some serological and epidemiological data on the viruses recovered from patients with subacute thyroiditis De Quervain. Med Microbiol Immunol 1975; 165 : 133-44.

3. Debons-Guillemin MC, Valla J, Gazeau J, W'ybier-Franqui J, Giron ML, Toubert ME, Canivet M, Périès J. No evidence of spumaretrovirus infection markers in 19 cases of De Quervain's thyroiditis. Aids Res Hum Retrovir $1992 ; 8: 1547$.

4. Lagaye S, Vexiau P, Morozov V, GuénebautClaudet V, Tobaly-Tapiero J, Canivet M, Cathelineau (;, Périès J, Emanoil-Ravier R. Human spumaretrovirus-related sequences in the DNA of leukocytes from patients with Graves disease. Proc Natl Acad Sci USA 1992; 89: 10070-4.

5. Bothe K, Aguzzi A, Lassmann H, Rethwilm A, Horak l. Progressive encephalopathy and myopathy in transgenic mice expressing human foamy virus. Science $1991 ; 253: 555-7$. 6. Saib A, Canivet $\mathrm{M}$, Giron ML, Bolgert $\mathrm{P}$, Valla J, Lagaye S, Périès J, de Thé H. Human foamy virus infection in myasthenia gravis. Lancet 1994 343: 666
La transplantation cellulaire dans les maladies hépatiques. On sait depuis longtemps que le foie, organe pourtant très différencié, est susceptible de régénération : le modèle le plus classique est l'hépatectomie des deux-tiers, où la régénération est assurée par une ou deux divisions d'hépatocytes différenciés. Un autre modèle, proposé par l'équipe de Brinster à Philadelphie, (PA, USA), fait appel à des souris transgéniques pour une construction génique hépatotoxique albumine-urokinase (Alb-uPA), ciblée vers le foie. Le transgène crée un déficit hépatique fonctionnel tel qu'il constitue une stimulation permanente de croissance hépatique. Les quelques hépatocytes qui se débarrassent par hasard du transgène délétère ont une expansion sélective qui leur permet de remplacer complètement le foie malade en huit à dix semaines $(\mathrm{m} / \mathrm{s}$ $n^{\circ} 2$, vol. 9, p. 230, [1]). Cette régénération procède-t-elle à partir de «cellules souches" hépatiques? Ou les hépatocytes complètement différenciés ont-ils une capacité de division suffisante pour remplacer complètement un foie défaillant? La réponse à cette question est apportée par la même équipe [2] : les auteurs ont injecté dans la rate de souris transgéniques Alb-uPA des hépatocytes issus de souris elles-mêmes transgéniques pour diverses constructions géniques permettant le repérage ultérieur des cellules hépatiques transplantées et leurs filles. Parmi les cellules injectées qui migrent dans le foie $(3,5 \%$ à $17 \%)$ une proportion importante développent des clones de cellules hépatiques, fonctionnelles de surcroît: après 4 à 6 semaines, jusqu'à $80 \%$ du foie est remplacé par des cellules issues des hépatocytes transplantés; ces cellules se montrent capables d'au moins douze divisions. Les auteurs n'ont pas vu se développer de tumeur pendant les six mois de l'étude, mais les hépatocarcinomes qu'ils ont observés dans leur étude précédente apparaissaient au bout de huit mois $\left(\mathrm{m} / \mathrm{s} n^{\circ} 2\right.$, vol. 9 , p. 230). Ces résultats ont plusieurs implications: s'ils sont confirmés avec des hépatocytes humains, la transplantation de cellules hépatiques humaines au cours de la destruction fulminante du foie pourrait être envisagée; la correction d'un déficit métabolique par thérapie génique à l'aide d'hépatocytes modifiés génétiquement est sans doute possible. Mais il faudrait que les conditions de croissance de ces hépatocytes fussent préservées, soit par destruction hépatique associée, soit par apport des facteurs de croissance nécessaires.

[1. Sandgren EP et al. Cell 1991; 66: 245-56.]

[2. Rhim JA et al. Science 1994; 263: 1149-52.]

口[ Épilepsie partielle (frontale), une maladie autosomique dominante. De nombreuses épilepsies ont une composante génétique, mais on ne connaissait pas encore d'épilepsie humaine, monogénique et partielle. Une équipe australienne (Melbourne) vient de décrire un syndrome épileptique familial, responsable de brèves crises motrices, nocturnes et répétées, ayant entraîné, chez la plupart des malades, une erreur de diagnostic (cauchemars, terreurs nocturnes, hystérie...) [1]. Six familles, australiennes et canadiennes, forment le coeur de la description, avec trenteneuf malades faisant des crises partielles, sans anomalie électrique. L'analyse de la ségrégation suggère fortement une transmission autosomique dominante. Il existe un modèle murin d'épilepsie partielle débutant dans l'hippocampe, autosomique dominante chez la souris E1. Le gène responsable de cette maladie a été localisé au chromosome 9 [2], ce qui fait rechercher le gène candidat de l'épilepsie humaine sur le chromosome 3 (si la synténie est conservée).

[1. Scheffer et al. Lancet 1994; 343: 515-7.]

[2. Rise ML et al. Science 1991; 2543: 669-73.]
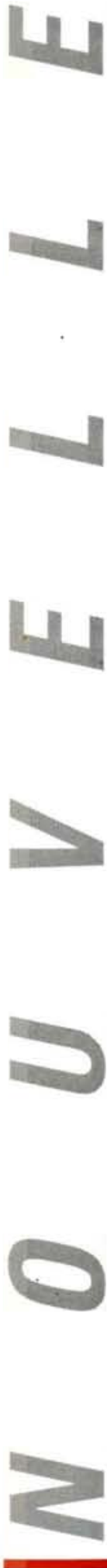